\title{
Costos y rendimientos en la labranza convencional y mecanizada de la agricultura familiar en la comunidad campesina Barrio Bajo de Matucana.
}

\section{Costs and performance in the conventional and mechanized tillage of family farming in the peasant community of Barrio Bajo in Matucana}

\author{
Catherine Alva ${ }^{1}$, Noriyuki Baba², José Velásquez ${ }^{3}$ \\ 1 Universidad Nacional Agraria La Molina, La Molina s/n, Lima, PE. \\ 2 Japan International Cooperation Agency - JICA, Apartado Postal 18-0261, Lima 18, PE. \\ ${ }^{3}$ Agro Rural, Jesús María, Lima 11, PE.
}

DOI: https://doi.org/10.33017/RevECIPeru2015.0021/

\section{Resumen}

En la labranza de la agricultura familiar de la sierra peruana convencionalmente se emplean aperos manuales (yunta y barreta). Actualmente no se tienen determinados los costos y rendimientos de trabajo de la labranza convencional y mecanizada. En consecuencia, esta investigación buscaba determinar cuántos son los costos y rendimientos de trabajo que el agricultor familiar invierte; a la vez, cuán adaptable es la mecanización según sus necesidades. Se verificó cuantitativamente que la labranza convencional tiene mayores costos y menores rendimientos que la labranza mecanizada, asimismo que esta última es adaptable a las necesidades que el agricultor familiar de la sierra peruana necesita. Para ello se evaluó el acceso desde la carretera a la parcela y la labranza en cada una de ellas (parcelas en laderas, terrazas y andenes) con cada tipo de apero (yunta, barreta y motocultores), tanto en tiempos, longitudes, esfuerzo y calidad del suelo labrado. El lugar donde se realizaron las evaluaciones fue la comunidad campesina Barrio Bajo de Matucana desde julio del 2,014 hasta enero del 2,015. Se determinó que el agricultor invierte con la branza convencional alrededor de S/.1,300 por hectárea con rendimientos alrededor de 440 metros cuadrados por día, mientras que de forma mecanizada alrededor de S/.700 por hectárea y 990 metros cuadrados por día. La labranza mecanizada beneficia al agricultor familiar por su menor costo y mayores rendimientos, además porque cubre sus necesidades. Sin embargo, aún falta evaluar (de forma mecanizada) con otros implementos para poder cubrir las demás etapas de la producción agrícola.

Descriptores: agricultura familiar, aperos manuales, motocultores, mecanización, costos, rendimientos.

\section{Abstract}

In tillage of family farming in the Peruvian highlands conventionally hand tools (yoke and barreta) are used. Currently, the costs and the work performance of conventional and mechanized farming are not certain. Consequently, this research seeks to determine how much are the costs and work performance which the family farmer invests; at the same time, how adaptable is the mechanization to their needs. It was verified quantitatively that tillage has higher costs and lower returns than mechanical tillage, the latter is also adaptable to the needs of family farmers in the Peruvian highlands. For this, the road access to the land and farming was evaluated, in each one (plots on hillsides, terraces and platforms) with each type of implement (yoke, barreta and cultivators), both times, lengths, effort and tilled soil quality. The evaluations were performed was in the peasant community of Barrio Bajo Matucana from July 2,014 to January 2,015. It was determined that the farmer invests on conventional tillage about S/. 1,300 per hectare yields about 440 square meters per day, while the mechanized way is about $\mathrm{S} / .700$ per hectare and 990 square meters per day. Mechanical tillage 
benefits the family farmer for its lower cost and higher yields, as well as covering their needs. However, there is still evaluating (mechanized) with other implements to cover other stages of agricultural production.

Keywords: Family farming, manual tools, cultivators, mechanization, costs, performance.

\section{Introducción}

La agricultura familiar de América Latina y el Caribe aporta con el 80 por ciento de las explotaciones agrícolas y genera el 70 por ciento del empleo agrícola en la región, es por ello que la FAO declaró al año 2,014 como "Año Internacional de la Agricultura Familiar". Asimismo, indicó que a medida que se reduzcan los altos costos de transacción del sector, los productores incrementarán su inversión y producción, combinando la sabiduría ancestral de los agricultores familiares con los desarrollos tecnológicos modernos [1].

En el Perú, la agricultura familiar se desarrolla en áreas menores a 10 hectáreas. De esta manera, según M. Pintado, basándose en el Cenagro 2012, mostró que son 1'392,032 los productores familiares de la sierra, es decir que el 65 por ciento de los productores familiares se encuentran en la sierra peruana [2]. Por su parte, F. Eguren indicó que la agricultura familiar del Perú contiene el 75 por ciento de las tierras cultivadas [3]. Además, PAF señaló que la agricultura familiar provee el 80 por ciento de los alimentos consumidos a nivel nacional y, en especial, genera el 79 por ciento del empleo agrícola en el país, es decir emplea a 3 millones de personas [4].

Por su lado, N. Baba determinó que la superficie agrícola promedio por productor familiar es de 1.9 ha en la sierra peruana [5]. Además, a partir del IV Cenagro del INEI se determinó que el 66 por ciento utiliza animales para realizar trabajos agrícolas 0 pecuarios, mientras solo el 21 por ciento utiliza tractores [6].

Lo explicado ratifica la importancia de la agricultura familiar, no sólo en la alimentación, sino en la generación de empleo; sin embargo, no existe información relacionada a los rendimientos de trabajo y los costos que le genera al productor familiar. En la producción de cultivos se necesita preparar el suelo para la siembra mediante la labranza, luego de realizada la siembra siguen actividades de mantenimiento del cultivo hasta la cosecha y posterior destino que puede ser el auto consumo o la venta. La labranza consta de la roturación y el mullido, estas actividades son las que demandan mayor esfuerzo y mano de obra, por lo cual son los varones quienes generalmente se dedican a esta etapa de la producción agrícola, delegando a la mujer el mantenimiento del cultivo.

No se cuenta con los costos ni con los rendimientos de trabajo de las etapas de la producción agrícola de la agricultura familiar a nivel nacional, es por ello que el objetivo fue determinar los costos y rendimientos de trabajo de la labranza, actividad que demanda de mayor mano de obra e inversión de dinero en la agricultura familiar. Además, como la labranza convencional de la agricultura familiar se realiza con aperos manuales, es decir yunta y barreta, se quiso compararlos con la forma mecanizada en sus rendimientos y costos. Sin embargo, como esta agricultura se partica en áreas promedio de 1.9 ha y en zonas de ladera, terrazas 0 andenes, se determinó realizar el estudio con motocultores.

\section{Metodologia}

\subsection{Materiales}

Para las evaluaciones de la labranza se emplearon aperos manuales, yunta y barreta, y mecanizados, motocultores Shifeng SF121 (14.7 Hp), Honda FJ500 (4.8 Hp) y Honda FF500 (4.5 Hp). Para las mediciones y la evaluación de la calidad de la labranza se utilizaron cronómetros, oxímetro de pulso, navegador GPS, probetas, balanzas, winchas, tamices, jalones, niveles, muestreadores de densidad y palas.

\subsection{Ubicación del área estudio}

La ubicación donde se realizaron las evaluaciones fue en la comunidad Barrio Bajo de Matucana, provincia de Huarochirí-Lima, en los lugares de Shilco y Matara. En la Tabla 1 se muestran sus altitudes y temperaturas.

Tabla 1: Lugares donde se realizaron as evaluaciones, altitud y temperatura.

\begin{tabular}{|c|c|c|}
\hline Lugar & Altitud $(\mathrm{msnm})$ & Temperatura $\left({ }^{\circ} \mathrm{C}\right)$ \\
\hline Shilco & 2,700 & \multirow{2}{*}{$18-8$} \\
\hline Matara & 3,000 & \\
\hline
\end{tabular}

\subsection{Procedimiento}


Las evaluaciones se iniciaron con la determinación de las áreas de las parcelas y las distancias de acceso, utilizándose para ello las winchas y GPS. Se midió al inicio de las actividades la frecuencia cardíaca de cada operador (con el saturador de oxígeno) y cada apero fue trasladado por los operadores desde la carretera hacia la parcela; se contabilizó la cantidad de operadores que fueron necesarios para el traslado de cada apero y los tiempos (con los cronómetros) que demoraron en el acceso. Seguidamente, se procedieron con las evaluaciones de los aperos en las parcelas, se midieron los tiempos de trabajo, las veces que se tuvo que realizar el trabajo (roturación y/o mullido), las profundidades de la labranza, el tamaño de la partícula desmenuzada (con los tamices), la densidad aparente del suelo (con los muestreadores cilíndricos) y el consumo de combustible (con las probetas).

Las evaluaciones culminaron con el retorno de los aperos hacia la carretera, donde también se midieron los tiempos del traslado, la cantidad de operadores necesarios y sus frecuencias cardíacas.

\section{Resultados y discusión}

\section{1 Área de estudio}

Las áreas donde se realizaron las evaluaciones fueron en ladera, terrazas y andenes. El tamaño total disponible del área se dividió en cinco parcelas para que los aperos trabajen en simultáneo. En la Tabla 2 se muestran las áreas (en metros cuadrados) que fueron evaluadas.

Tabla 2: Parcelas evaluadas, tipo y área.

\begin{tabular}{|c|c|}
\hline Parcelas & Área $\left(\mathrm{m}^{2}\right)$ \\
\hline Matara (1 y 2) - Laderas en descanso & 877.5 \\
\hline Matara (10 y 11) - Terrazas en descanso & 633.5 \\
\hline Shilco (1, 2, 3 y 4) - Andenes con rastrojo & 608.8 \\
\hline
\end{tabular}

El área total donde se evaluó la labranza en ladera fue de $877 \mathrm{~m}^{2}$, en terrazas fue de $633.5 \mathrm{~m}^{2}$ y en andenes fue de $608 \mathrm{~m}^{2}$. Además, "descanso" indicó que la parcela estaba con kikuyo (con cobertura y difícil de labrar) y "rastrojo" en rotación de cultivo (sin cobertura y fácil de labrar).

\subsection{Número de tramos de los accesos}

El número de tramos en los accesos dependió de las irregularidades de los mismos. En la Tabla 3 se muestra el número de tramos de los accesos a las parcelas. Se observa que no hay una relación directa entre el número de tramos con la distancia de acceso puesto que ello dependió de cuán irregular fue el terreno. Es decir, que los accesos más irregulares fueron los de Matara 10 con 13 tramos y Shilco $1_{\text {bajada }}$ con 7 tramos.

Tabla 3: Número de tramos y distancia de los accesos.

\begin{tabular}{|c|c|c|c|}
\hline Parcela & Tipo & Distancia $(\mathrm{m})$ & Tramos \\
\hline Matara 1 & Ladera & 145.7 & 7 \\
\hline Matara 2 & Ladera & 128 & 4 \\
\hline Matara 10 & Terrazas & 265.8 & 13 \\
\hline Shilco $1_{\text {baja }}$ & \multirow{2}{*}{ Andenes } & 366.4 & 7 \\
\cline { 1 - 3 } & & 152 & 6 \\
\hline
\end{tabular}

\subsection{Tiempo y velocidad de acceso}

Los tiempos de acceso de la carretera a la parcela tanto de subida como de bajada fueron medidos y con ello se calcularon los promedios de la velocidad de tramos en metros por segundo y el tiempo total en segundos por 100 metros lineales.

En la Figura 1 se muestran los resultados para las parcelas en Matara 1, de esta manera se determinó que el apero Manual fue el más rápido con un tiempo de 93 segundos por $100 \mathrm{ml}$ en el acceso de bajada y 112.6 segundos por $100 \mathrm{ml}$ para la subida. Mientras que, el apero menos rápido fue el motocultor SF121 con 923 segundos por $100 \mathrm{ml}$ para el acceso de bajada y $1,367.7$ segundos por $100 \mathrm{ml}$ para la subida. 


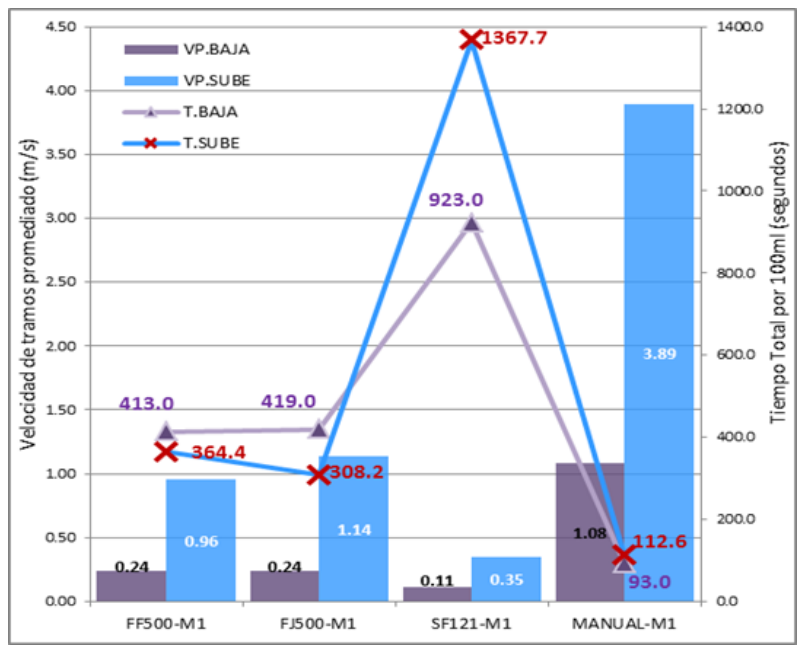

Figura 1: Velocidad de tramos promedio y Tiempo total por $100 \mathrm{ml}$ del acceso de Matara 1.

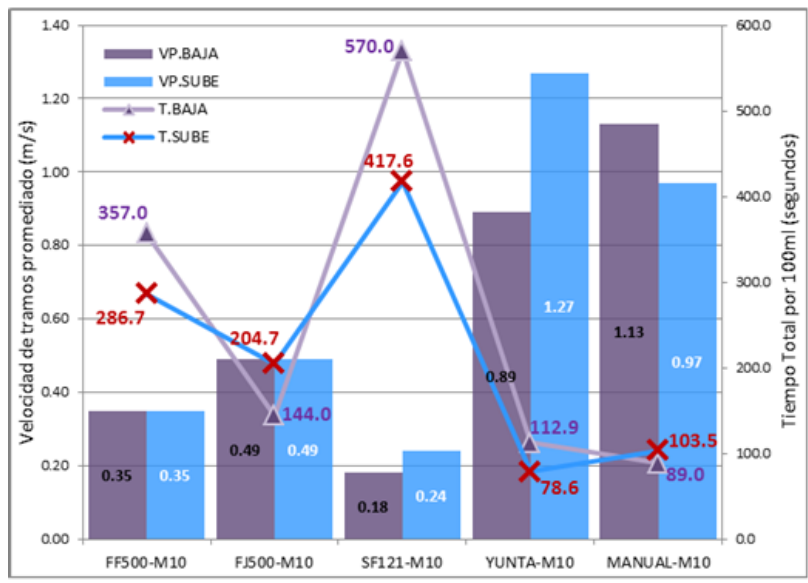

Figura 2: Velocidad de tramos promedio y Tiempo total por 100ml del acceso de Matara 10.

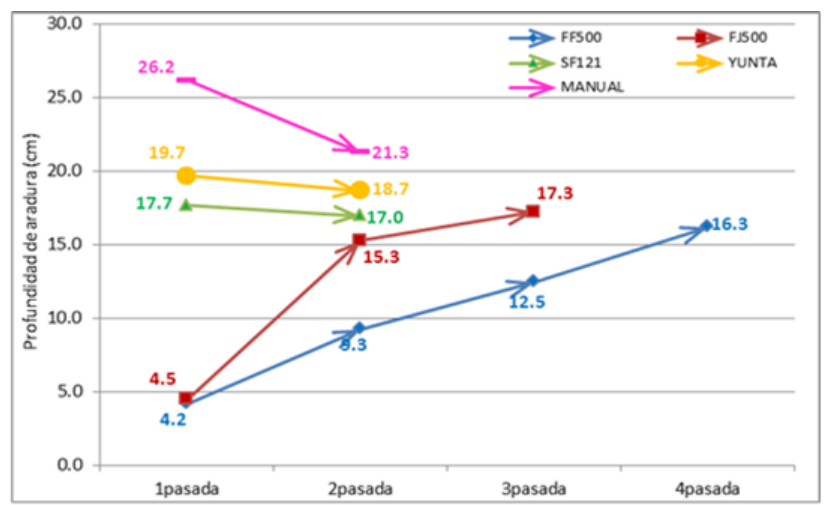

Figura 3: Profundidad promedio de labranza en Matara 10.

Para el acceso de Matara 10 se evaluaron los dos tipos de aperos manuales, Yunta y Manual (barreta), y los tres motocultores. En la Figura 2 se observa que el más rápido resultó ser Manual en la bajada y Yunta en la subida, con 89 y 78.6 segundos por $100 \mathrm{ml}$, respectivamente. Mientras que dentro de los motocultores el modelo FJ500 fue quien resultó ser el más rápido con 144 y 204.7 segundos por $100 \mathrm{ml}$, en la bajada y subida, respectivamente.

Además, para el traslado del motocultor SF121 se requirió entre 4 a 6 operarios, mientras los otros dos motocultores necesitaron solo de 2 operarios, 
cada uno. Para la Yunta se necesitó de un operario y una acémila; mientras que, para Manual, cada operario cargó por sí mismo su barreta, desde la carretera a la parcela.

Con estos resultados se determinó que dada las irregularidades del terreno de los accesos los aperos manuales están mejor adaptados para este medio, demandando alrededor de 100 segundos por cada $100 \mathrm{ml}$. Asimismo, los motocultores presentan importante desventaja si son muy grandes como el motocultor SF121 (14.7Hp), aunque moderadamente aceptables para los motocultores de pequeñas potencias como FJ500 $(4.8 \mathrm{Hp})$ y FF500 $(4.5 \mathrm{Hp})$.

\subsection{Profundidad de labranza}

La profundidad de labranza fue medida después de cada pasada (roturación y/o mullido). En la Figura 3 se muestran los resultados de la profundidad promedio medida en centímetros de las parcelas labradas en Matara 10, terraza en descanso. Se podrá observar que los aperos manuales (Yunta y Manual) sólo realizaron dos pasadas (roturado y mullido), mientras los motocultores realizaron entre dos a cuatro pasadas (solo mullido). Para estas parcelas de terrazas en descanso con kikuyo, quien obtuvo mayor profundidad en la última pasada fue Manual (barreta) con $21.3 \mathrm{~cm}$, seguido de Yunta con $18.7 \mathrm{~cm}$, y entre los motocultores quien logró mayor profundidad fue FJ500 con $17.3 \mathrm{~cm}$.

En la Figura 4 se muestran los resultados de las profundidades en Shilco, andenes con rastrojo (sin kikuyo). Se observó que para este caso a lo más se realizaron 2 pasadas y quien logró mayor profundidad fue el motocultor SF121 con $23.1 \mathrm{~cm}$ seguido de Yunta con $21.5 \mathrm{~cm}$ y FJ500 con 20.7 $\mathrm{cm}$.

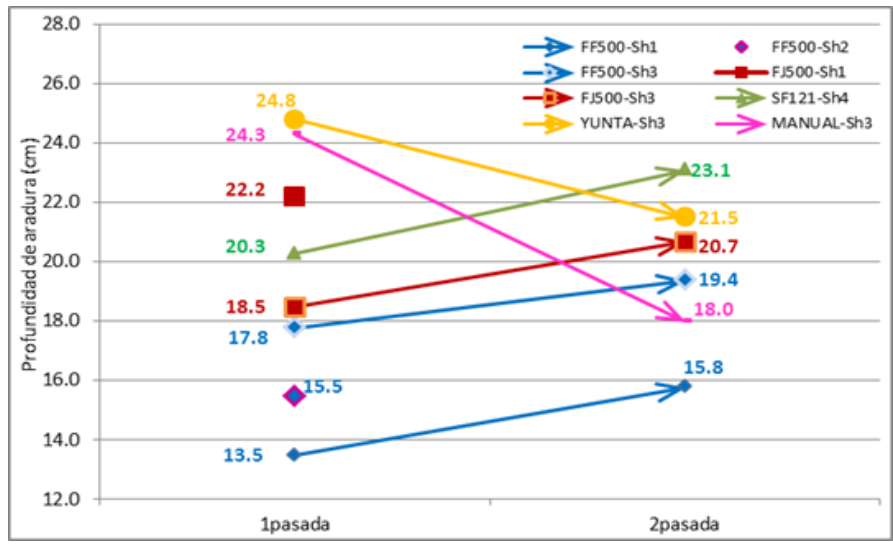

Figura 4: Profundidad de labranza en Shilco.
La profundidad máxima de labranza que requiere el agricultor familiar de Barrio Bajo es de $15 \mathrm{~cm}$ a $20 \mathrm{~cm}$ para la siembra. Es decir, que con todos los aperos se logró la profundidad necesaria para la siembra.

\subsection{Diámetro de partícula desmenuzada}

Los diámetros de partícula desmenuzada fueron determinados en cada pasada, es decir por cada vez que se labró sobre un área determinada. En la Figura 5 se muestran los resultados del diámetro de partícula para Matara 1. Se observó que como los aperos manuales (Yunta y Manual) siempre realizan dos pasadas, en la primera hicieron el roturado del suelo y por ello el tamaño de partículas es grande, y en la segunda realizaron el mullido por lo cual el tamaño de partícula disminuyó. Mientras que los motocultores provistos de azadas siempre mullen, por ello desde la primera pasada el diámetro de partícula es pequeño.

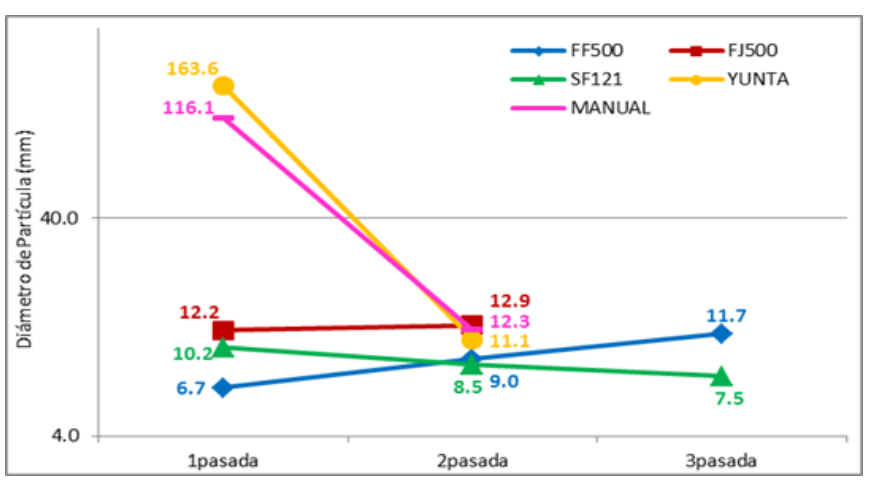

Figura 5: Diámetro promedio de partícula desmenuzada en Matara 1.

En la Figura 5, para los aperos manuales en la primera pasada resultaron diámetros promedio alrededor de $120 \mathrm{~mm}$, mientras que en la segunda alrededor de $12 \mathrm{~mm}$. Asimismo, para los motocultores los diámetros promedio resultaron entre $6.7 \mathrm{~mm}$ (primera pasada de FF500) a $12.7 \mathrm{~mm}$ (segunda pasada de FJ500).

Los resultados anteriores son para parcelas en ladera y descanso (con kikuyo), mientras que para parcelas en andenes y con rastrojos los diámetros por pasada resultaron diferentes como se muestra en la Figura 6. En ésta se observan que todos los aperos realizaron hasta dos pasadas como máximo por ser un suelo más fácil de labrar. De este modo, los aperos manuales durante la primera pasada obtuvieron $8.9 \mathrm{~mm}$ y $10.5 \mathrm{~mm}$, y en la 
segunda $8.5 \mathrm{~mm}$ y $11 \mathrm{~mm}$, para Yunta y Manual, respectivamente.

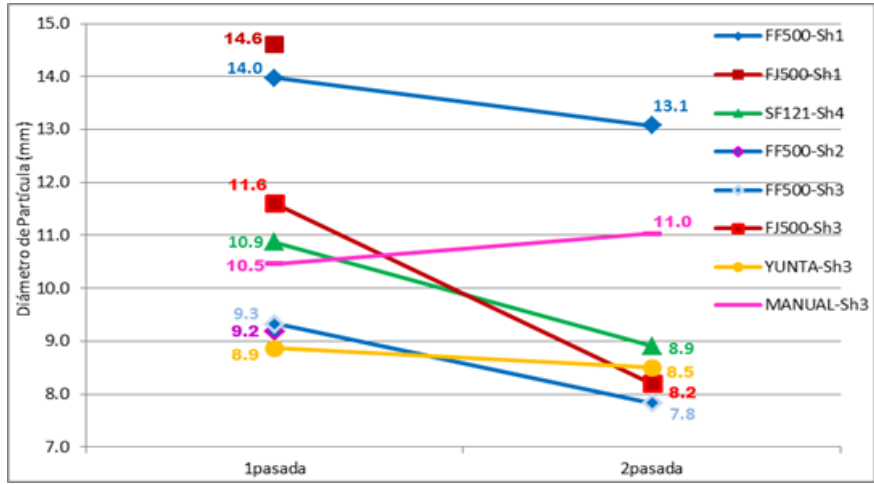

Figura 6: Diámetro promedio de partícula desmenuzada en Shilco.

Además, se observa en la Figura 6 que los motocultores siempre obtuvieron diámetros pequeños de partícula, de esta manera quien obtuvo menor diámetro culminada la labranza fue FF500 con $7.8 \mathrm{~mm}$ y FJ500 con $8.2 \mathrm{~mm}$.

Sin embargo, aunque los aperos manuales siempre realizaron dos pasadas pudieron obtener un diámetro menor a $20 \mathrm{~mm}$ que es lo deseable, en promedio. Además, aunque los motocultores pueden emplear más de dos pasadas, en cada una también obtuvieron diámetros de partículas menores a $20 \mathrm{~mm}$.

\subsection{Costos y Rendimiento de trabajo}

Los costos y rendimientos de trabajo resultaron diferentes tanto para parcelas en laderas y terrazas en descanso (Matara 1, 2, 10 y 11) como en parcelas en andenes con rastrojos (Shilco 1, 2, 3 y 4). La diferencia radicó en la dificultad de labrar suelo en descanso con kikuyo como las de Matara, y a la facilidad de labrar el suelo cuando está sin kikuyo, como las de Shilco; es decir, que el kikuyo es el principal problema en la labranza del agricultor familiar porque sus raíces son profundas y duras de desarraigar concentrándose en los primeros $10 \mathrm{~cm}$ de profundidad de suelo, además de ser resistente e invasivo.

En la Figura 7 se muestran los costos (barra roja: costo operativo; barra azul: costo de mano de obra; y línea negra punteada: costo total) y rendimientos en tiempo de trabajo (línea verde) en laderas (Matara 1 y 2) y terrazas (Matara 10 y 11) en descanso con kikuyo. De acuerdo a los costos de labranza es recomendable para parcelas de ladera en descanso labrar con el motocultor FF500 en
Matara 1 con S/.622 por hectárea; mientras que para terrazas en descanso labrar con FJ500 en Matara 10 con S/.927 por hectárea. Los mayores rendimientos de trabajo se obtuvieron en los mismos casos que para los costos, es decir 0.118 hectáreas por día (1,180 m2/día) con FF500 en Matara 1 y 0.057 hectáreas por día (570 m2/día) para FJ500 en Matara 10.

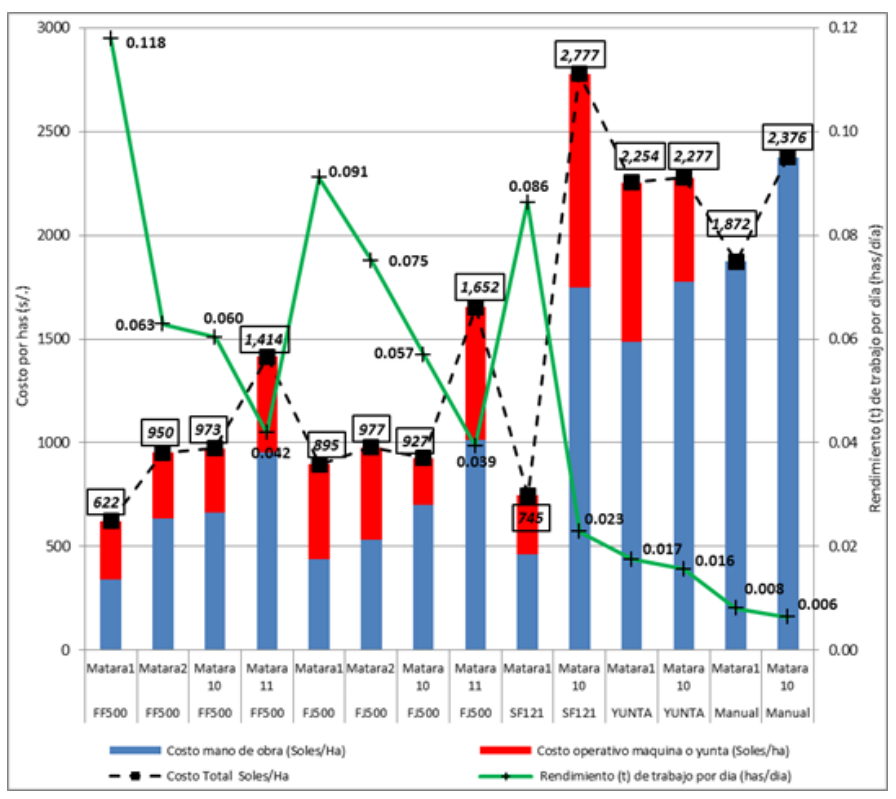

Figura 7: Costos y Rendimientos (t) de trabajo en Matara 1, 2, 10 y 11.

En la Figura 8 se muestran los costos (barra roja: costo operativo; barra azul: costo de mano de obra; y línea negra punteada: costo total) y rendimientos de trabajo (línea verde) en andenes con rastrojos (sin kikuyo). De acuerdo a los costos de labranza son recomendables los motocultores SF121 en Shilco 4 con S/.417 por hectárea y FF500 en Shilco 2 con S/.422 por hectárea. Asimismo, para mayores rendimientos de trabajo FF500 en Shilco 2 con 0.202 hectáreas por día $(2,020 \mathrm{~m} 2 /$ día $)$, FF500 en Shilco 1 con 0.156 hectáreas por día (1,560 m2/día) y SF121 en Shilco 4 con 0.145 hectáreas por día (1,450 m2/día).

Con los resultados de costos y rendimientos de trabajo se determinó que hay mucho beneficio en trabajar de la forma mecanizada en especial por el ahorro del costo de mano de obra que en la Figura 7 y la Figura 8 están representadas por las barras de color azul; pero a ello hay que añadirle las necesidades del agricultor familiar, en especial la de desarraigar el kikuyo del suelo cuando la parcela está en descanso, para lo cual es necesario roturar el suelo, no obstante dicha 
actividad lo realizan los aperos manuales mas no motocultores con azadas.

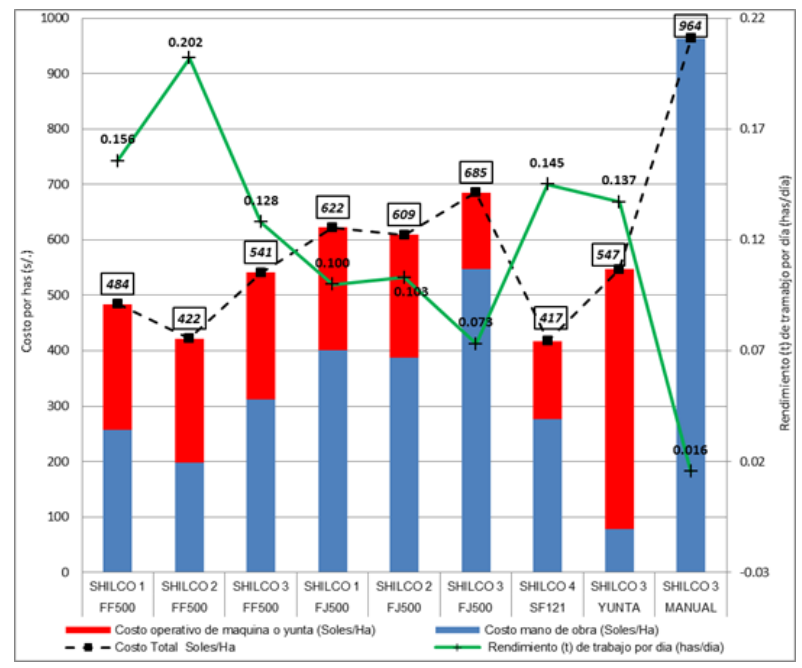

Figura 8: Costos y Rendimientos ( $t$ ) de trabajo en Shilco 1, 2, 3 y 4.

\section{Conclusiones}

Al comparar los costos y el rendimiento de trabajo de labrar entre la forma convencional y la mecanizada, resulta beneficioso para el agricultor familiar de la zona altondina la labranza con motocultores (mecanizada) en especial por el ahorro del costo de mano de obra y los mayores rendimientos en hectáreas por día que obtiene.

La mecanización de la labranza en las parcelas de laderas, terrazas y andenes se adapta a estas condiciones, aunque presenta limitaciones, de esta manera para el acceso desde la carretera a las parcelas es conveniente motocultores de mediana potencia, como FJ500 y FF500; mientras que para el trabajo de la labranza con los motocultores de pequeña potencia (FF500 de $4.8 \mathrm{Hp}$ y FJ500 de $4.5 \mathrm{Hp}$ ) resultan en la mayoría de casos ser más prácticos que con el motocultor de mayor potencia (SF121 de 14.7Hp). Empero, solo para suelos en descanso, debido a que el productor familiar necesita retirar el kikuyo no basta la labranza de motocultores con azadas, sino habría la necesidad de roturar el suelo previamente antes de hacer el mullido con las azadas de los motocultores por lo que también se necesita probar con otros implementos acoplados a éstos, por ejemplo, una reja.

La calidad de suelo labrado de forma mecanizada cubre las necesidades para la siembra en cuanto al tamaño de partícula y profundidad de suelo labrado que necesita el agricultor familiar.
Al agricultor le conviene producir en parcelas que estén en rotación de cultivos o con rastrojos, es decir, que no estén en descanso porque se desarrolla el kikuyo; este factor aumenta considerablemente los costos totales a la vez que disminuyen los rendimientos de trabajo por la dificultad de la labranza. Sin embargo, la etapa de descanso resulta importante en la recuperación de los minerales y la materia orgánica del suelo (la fertilización); por lo que aún hay un tema importante por analizar además de la mecanización en la producción de la agricultura familiar.

\section{Agradecimientos}

La realización de este trabajo ha sido posible por la unión de esfuerzos entre Agro Rural, Honda del Perú y la Agencia de Cooperación Internacional del Japón (JICA), con el apoyo de la Dirección Zonal Lima y la Universidad Nacional Agraria La Molina. Agradecemos a los productores de la comunidad campesina Barrio Bajo de Matucana por haber participado en las evaluaciones.

\section{Referencias}

[1] FAO (2013). Centro de noticias ONU. Consultado 08 de nov. 2015. Formato HTTP disponible en http://www.un.org/spanish/News/story.asp?n ewsID=26019\#. VkADibcve00

[2] M. Pintado (2014). Los agricultores familiares peruanos: ¿cuánto son? Y ¿dónde están? (en línea). La Revista Agraria. (168): pp. 4-6. Consultado 08 de nov. 2015. Formato PDF. Disponible en

http://www.larevistaagraria.org/sites/default/fil es//revista/LRA168/Agrodata.pdf

[3] F. Eguren (2014). El necesario reencuentro con la agricultura familiar (en línea). La Revista Agraria. 15 (163): pp. 3-4. Consultado 12 may. 2015. Formato PDF.

Disponible en

http://www.larevistaagraria.org/sites/default/fil es//revista/LRA163/LRA-163.pdf

[4] PAF (2014). ¿Qué es la agricultura familiar? Declaración por la agricultura familiar. Semana de la agricultura familiar (en línea). 1 ed. Jun. 2014: p. 16. Consultado 17 jun. 2015. Formato PDF. Disponible en http://www.observatorioseguridadalimentaria. org/sites/default/files/publicaciones/archivos/

$$
\begin{aligned}
& \text { Boletin por ciento20PAF } \\
& \text { por }
\end{aligned}
$$

ciento20final.compressed_0.pdf 
[5] N. Baba (2015). Comparación de la política de terrazas entre Perú y Japón. Eds. TILLMANN, T. y BUENO DE MEZQUITA, M. II Congreso Internacional de Terrazas: encuentro de cultura y saberes de terrazas del mundo. 1 ed. Cusco, PE, CBC/JICA. 395 pp. 354-365 p. Disponible (también) en: http://www.infoandina.org/sites/default/files/p ublication/files/ii congreso terrazas pdf res ol baja.pdf
[6] INEI (2012). IV Censo Nacional Agropecuario 2012 (en línea). Consultado 25 jun. 2015. Formato HTML. Disponible en http://censos.inei.gob.pe/Cenagro/redatam/\#

E-mails: 20070277@lamolina.edu.pe ww3yuki2000@yahoo.co.jp, josevelasquez_m@hotmail.com 